\section{Going through the changes}

\author{
Mark Johnson
}

On the Nature of Human Plasticity. By Richard M. Lerner. Cambridge University Press: 1984. Pp.208. £17.50, \$29.95.

"Plasticity" is one of those labels that has taken on a variety of different, but overlapping, meanings. For example, the neurophysiologist may use the term to refer to the capacity of the nervous system to regenerate or reorganize after injury or environmental change, while some psychologists choose to reserve the term for the possible range of variation that can occur in individual development. Because of this confusion of meanings, it is essential that anyone setting out to integrate information on "plasticity" from various disciplines (in this case genetics, neurobiology, evolution, individual and group psychology) should adopt a clear and precise definition. In his book, however, Richard Lerner appears to be content to allow his interpretation of the word to emerge from the interaction between the reader and the 174 pages of text. This is both a strength and a weakness. While it enables the author to apply the term to everything from molecular genetics to social relationships, the price to be paid is the rather doubtful usefulness of his conception within some levels of explanation.

Two main theses with regard to human development are put forward in the book. First, Lerner suggests that "plasticity" is not confined to the early years but exists throughout the entire life-span. The "lifespan" developmental view is a recent school of thought in psychology which emphasizes the capacity for change in the adult. This emphasis on the adult allows its proponents, including Lerner, to use "plasticity" to refer to any changes in structure or function in the organism. However, when this conception of the term is confused with "plasticity" as a specific response to the environment, which it is in the book, the reader is left with the implication that all developmental change is environmentally specified. And this while claiming to have moved beyond the old nature-nurture debate.

The second main thesis is that variables and processes at different levels (biological, individual psychological, dyadic, social and so on) may contribute to human functioning at any one time. These levels do not work independently, in parallel, but are reciprocally influential. Each level is seen to be "embedded" within the others. Lerner formalizes this idea in a framework for describing the development of individuals within their multilevelled environment. However, as he himself admits, this framework merely constitutes a specification of the domain

within which future theory and research about individual development should be generated. Its usefulness can only be assessed by the success or otherwise of the models it gives rise to. Even if successful at the psychological level, it is not clear whether such a framework would be appropriate at, for example, the neurobiological level. Equivalence of terminology does not imply equivalence of mechanism.

Lerner sees the ideas presented in the book as being of consequence for the clinician. Since each "level" is engaged in dynamic interaction with the others in which it is embedded, it follows that intervention by the clinician at one level will have effects at other levels. For example, changing an individual's diet may have an influence on neurotransmitters which may then have effects on behaviour, and

\section{Nonsenses of today}

\section{Joseph Silk}

Perfect Symmetry: The Search for the Beginning of Time. By Heinz R. Pagels. Simon \& Schuster: 1985. Pp. 329. \$18.95. To be published in Britain later this year by Michael Joseph.

PARTICle physics and cosmology have developed a symbiotic relationship. While it would take a particle accelerator stretching from here to Alpha Centauri to smash particles together at energies approaching the grand unification scale (and we may safely consider this to be outside the future budgets of CERN or Fermilab), such energies were reached routinely in the very early Universe. Hence the attention being paid by particle theorists to constraints from cosmology. Already, many exotic theories have been eliminated, and others, phoenix-like, have arisen. Does the dark matter that pervades the visible Universe consist of massive chunks of quark matter at nuclear density? Or does it consist of shadow matter, a whole universe of stars and galaxies, invisible to us apart from their gravitational interaction?

This book by Heinz Pagels, a particle physicist, provides a lucid introduction to the interplay between cosmology and particle physics. He describes the motivation and basis for grand unification theories, and their role in cosmology, and includes a chapter on the inflation of the Universe, one of the most notable results of the synthesis between particle physics and cosmology. Inflationary cosmology provides a scientific basis for understanding why the Universe is so large, and why it is so isotropic (that is, similar in all directions). It explains why the Universe is almost, but not quite, uniform: from the primordial fluctuations in density, the galaxies eventually grew thence on dyadic and other social relationships. The effects at other levels may be beneficial or detrimental, but in order to predict them some understanding of the mechanisms of interaction between levels is required. Lerner offers us only description, and description is not adequate for prediction.

In his foreword, Paul Baltes states that despite his approval of the book he is convinced that the author will be criticized for his commitment to interdisciplinary breadth. On the contrary, such a commitment is to be applauded. However, the synthesis we are offered in this book is, like all mirages, attractive at a distance but elusive close up.

Mark Johnson is in the Medical Research Council's Cognitive Development Unit, 17 Gordon Street, London WCIH OAH, UK.

Although the hottest topics from last year's conference season, such as quark nuggets or shadow matter, are inevitably absent, there is a sufficiently solid introduction to the new physics that the reader will have little difficulty in making sense of the latest developments that appear in the semi-popular press. But to give him full credit, Pagels does not hesitate to tackle some vertiginous issues. What happened before the big bang? What was the origin of everything? Our Universe may well have begun as a quantum fluctuation: before, there was nothing, not even time or space, but absolutely nothing.

Much of the text is devoted to an account of the astronomer's Universe. The latest discoveries from space astronomy are described, ranging from the infrared-emitting embryo stars being hatched deep within dense interstellar gas clouds to the tenuous hot plasma glowing in X-rays throughout megaparsecs of intergalactic space in the great galaxy clusters. All of this, and a capsule description of the evolution of the big bang, from the beginning of the knowable Universe, some $10^{-43} \mathrm{~s}$ after the initial singularity, until about $1 \times 10^{10} \mathrm{yr}$ later.

The book is written for the intelligent layman, and thus non-technical and not unduly laden with jargon. It is, however, replete with quotations that help communicate the excitement at the frontier of science. My favourite is Eddington's reaction to the discovery of the first white dwarf star, Sirius B, supposedly made of material thousands of times denser than any known matter: "Shut up. Don't talk nonsense". Eddington's comment has been repeated many times since in reaction to novel observations or experimental data: seventy years later, it remains to be seen whether we have truly learned our lesson.

Joseph Silk is a Professor in the Department of Astronomy, University of California, Berkeley, California 94720 , USA. 